\title{
The Weak Coupling between MPS and BEM for Wave Structure Interaction Simulation
}

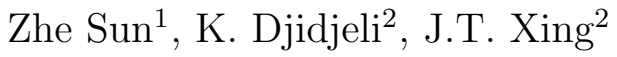 \\ ${ }^{1}$ School of Naval Architecture and Ocean Engineering, Dalian University of Technology, Dalian, China \\ ${ }^{2}$ CED/FSI, FEE, University of Southampton, Southampton, UK
}

\begin{abstract}
As a Lagrangian type meshless method, the MPS is suitable for violent free surface problems. In this paper, for problems where violent free surface deformation only occur in a constrained area, the efficiency of MPS is further improved by weak coupling with BEM. More specifically, the whole computational domain is modelled by BEM whereas the MPS model only covers the violent flow area. Since the computational time of BEM is negligible compared with the time required by MPS, the overall computational efficiency could be improved by this coupling scheme (depends on how much of the MPS domain is replaced by BEM). The MPS model is advanced by the information from BEM result at each time step up to the time when the free surface is about to break. The MPS solver will continue the simulation with the "old" BEM information just before breaking, based on the assumption that the flow change at the MPS-BEM interface area is small enough. The proposed scheme is validated by two problems and a relatively good accuracy is obtained by comparing with published results in the literature.
\end{abstract}

Keywords: MPS, BEM, Weak coupling, Wave Structure Interaction

\section{Introduction}

Particle method, compared to traditional CFD methods, is an emerging CFD tool for violent free surface flow problems. Due to its two main features i.e. meshless space discretization and Lagrangian type flow description, particle methods are very suitable for problems with large deformation of flow boundaries. More specifically, the meshless way of space discretization avoids the mesh distortion issue in traditional mesh-based approach, especially for problems with large boundary deformation like free surface flow. And also the Lagrangian type flow description makes it easier for tracking the rapidly moving boundaries. More detailed discussion about meshless methods could be found in ( $\mathrm{Li}$ and Liu, 2002, 2007; Zhang et al., 2003). As one of the representative particle method, the Moving Particle Semi-implicit (MPS) Method has been successfully applied to many violent free surface problems (Koshizuka and Oka, 1996; Sun et al., 2016, 2015; Xing, 2016). However, one of the problems of the particle methods is the high computational cost. Many researchers have proposed various approaches to improve the efficiency such as multi-resolution (Shibata et al., 2012), parallel computation (Duan and Chen, 2015) and hybrid solver (Sueyoshi et al., 2007) etc. In this paper, the hybrid solver approach will be discussed. 
Both of the Navier-Stokes and the potential flow models have their strengths and weaknesses (Sriram et al., 2014). The potential flow model can not handle the case where wave-breaking is involved. But it is usually more efficient and stable for large scale and long time simulation. The Navier-Stokes model (or often referred as CFD approaches) on the other hand is a general and robust solver that can simulate the violent and complicated free surface deformation cases. The disadvantages of the CFD approaches include the heavy computational cost and energy loss for long time simulation.

It is a logical choice to combine these two solvers for the problems in which the violent wave-breaking only occurs in small constrained area, and the flow motion in most of remaining area is relatively smooth. In a hybrid model, the whole computational domain is divided into sub-domains where different solvers are applied appropriately. Based on the way of exchanging information between these two solvers, the hybrid model could be classified as strong and weak couplings. A detailed review of the hybrid solver can be found in (Sriram et al., 2014; Grilli, 2008). A brief outline of the representative works about both the strong and weak couplings is given below.

In the strong coupling, the two solvers are mutually dependent on each other, i.e. the boundary values of each solver have to be calculated based on information from the other one. Both the mesh-based and meshless CFD methods have been tried to be incorporated with potential solvers for free surface flow problems. Colicchio et al. (Colicchio et al., 2006) combined a fixed-grid FD (Finite Difference) method (using VOF to capture the free surface) with BEM (Boundary Element Method) method to calculate various free surface flow problems. There is an overlap area between two sub-domains where the information is exchanged and consequently provide the necessary boundary condition to drive each solver. Grilli (Grilli, 2008) implemented the coupling in a different way in which the Navier-Stokes domain is fully submerged inside the potential flow domain and the later solver covers the whole computational domain. The dynamic pressure is included as a source term in the NavierStokes equation. For the coupling with meshless method, Sueyoshi et al. (Sueyoshi et al., 2007) used the MPS method to model the upper domain which includes free surface and BEM to simulate the area which is close to the bottom of tank. The information is exchanged on the interface where MPS particle moves in Lagrangian way and BEM boundary is fixed in space. Sriram et al. (Sriram et al., 2014) combined the FE based potential flow model with another particle method MLPG_R. The communication between the two solvers are conducted via a moving overlapping area. The velocity of the MLPG_R particle in this area is determined by linearly interpolating velocity from both solvers. The strong coupling strategy normally tends to be more accurate since some kind of convergence criteria is required during the exchanging of information. But for the same reason, several iterations will also have to be conducted, and consequently make the simulation more computational expensive.

The weak coupling strategy means one of the solvers covers the whole computational domain and provide the boundary condition for the other one during the whole simulation process without the need of any feedback from it. Normally it is the potential flow model, which acts as the "base" solver to initialize and drive the Navier-Stokes solver in the sub-domain where the violent free surface deformation occurs. The notable examples that involve the mesh-based CFD methods include the weak coupling between FVM (Finite Volume Method) and BEM for breaking of solitary wave on slop (C.Lachaume et al., 2003). The weak coupling between LBM ( Lattice Boltzmann method) and BEM were conducted for solving the same problem as well (C.F.Janssen et al., 2010). The particle method SPH has also been used to calculate the post-breaking waves incorporated with Boussinesq method (Kassiotis et al., 2011). The weak coupling strategy was investigated in this study using modified MPS (proposed by (Sun et al., 2015, 2016)) and BEM.

The remaining part of this paper is organised as follows: first, the numerical models that were used in the coupling, i.e. MPS and BEM methods are reviewed in section 2; then the weak coupling scheme between the two solvers is illustrated in section 3. In section 4, the proposed computation model is 
tested using two numerical examples, i.e. propagation of regular wave and breaking solitary wave impacting flexible walls before conclusions are drawn in section 5 .

\section{Numerical Models}

In this section, the numerical models used for the coupling, i.e. modified MPS and BEM methods, are briefly reviewed.

\section{$2.1 \quad$ Modified MPS method}

The modified MPS method developed by the authors (Sun et al., 2016, 2015) is used in this study. A brief illustration is given below.

\subsubsection{Governing equations}

The problems investigated here are the ones where the viscosity effect is quite small. As such, the Lagrangian form of incompressible and inviscid Navier-Stokes equations are employed here as the governing equations of the flow.

$$
\begin{aligned}
& \frac{D \mathbf{u}}{D t}=\frac{\mathbf{u}^{(k+1)}-\mathbf{u}^{(k)}}{\Delta t}=\mathbf{g}-\frac{\nabla p}{\rho_{0}} \\
& \nabla \cdot \mathbf{u}=0
\end{aligned}
$$

where $\mathbf{u}, p$ and $\rho_{0}$ are the fluid velocity, pressure and density, respectively. $\mathbf{g}$ is the vector pointing to the gravity direction, i.e. $\mathbf{g}=[0,-g]$, where $g$ is the value of gravity acceleration.

\subsubsection{Numerical implementation}

The above governing equation is solved by standard projection method (Chorin, 1967) as follows:

Firstly, the flow configuration is advanced to an intermediate state without considering pressure by:

$$
\begin{aligned}
& \mathbf{u}^{(*)}=\mathbf{u}^{(k)}+\Delta t \mathbf{g} \\
& \mathbf{r}^{(*)}=\mathbf{r}^{(k)}+\Delta t \mathbf{u}^{(*)}
\end{aligned}
$$

where $\mathbf{r}$ represents the location vector of particles. The superscript $*$ indicates the value of intermediate status of a particular time step.

Secondly, based on the velocity and particle distribution of the intermediate state, a pressure Poisson equation is then formulated as:

$$
\nabla^{2} p^{(k+1)}=\rho_{0} \frac{\nabla \cdot \mathbf{u}^{(*)}}{\Delta t}+\alpha \rho_{0} \frac{n_{0}-n^{(k)}}{n_{0} \Delta t^{2}}
$$

where $n$ indicate the distribution situation of particles, which is called "particle density". The subscript 0 corresponds to uniform particle distribution. The coefficient $\alpha$ in Poisson equation (Eq. (3)) 
is chosen in the way that there would be no further compression (or expansion) if the flow was being compressed (or expanded) in the last step.

For the solid boundary, the Neumann boundary condition is applied. On the free surface, the free surface particles will be identified and the zero pressure condition will then be imposed.

The gradient and Laplacian operators in the above Poisson equation (i.e. Eq. (3)) are discretized by a weighted average approach (Koshizuka and Oka, 1996). The resultant linear system is then solved by Generalized Minimum Residual (GMRES) method (Saad and Schultz, 1986).

Finally, after obtaining the pressure, the velocity and location are then updated as:

$$
\begin{aligned}
& \mathbf{u}^{(k+1)}=\mathbf{u}^{(*)}-\Delta t \frac{\nabla p^{(k+1)}}{\rho_{0}} \\
& \mathbf{r}^{(k+1)}=\mathbf{r}^{(k)}+\Delta t \mathbf{u}^{(k+1)}
\end{aligned}
$$

In order to improve the stability and accuracy, several numerical techniques have been developed for the modified MPS method. That includes the virtual particle technique that compensates the lack of particles outside solid boundary; corrected intermediate velocity that improves the velocity divergence calculation accuracy near solid boundary; a simple and accurate free surface particle identification method that reduces the pressure noise; particle shifting \& collision handling techniques that improve the regularity of particle distribution (hence improve the accuracy); and finally a more efficient particle searching strategy that requires only $2.5 / 9$ of the original searching area. The details of the implementation of these schemes could be found in (Sun et al., 2016, 2015).

\subsection{Boundary Element Method}

\subsubsection{Governing equations and boundary conditions}

As a well established method, there are several ways of implementing BEM. In this study, the approach described in (Grilli and Subramanya, 1996; Sun et al., 2012) are adopted.

Under the inviscid and irrotational assumption, the fluid velocity $\mathbf{u}$ could be expressed by the gradient of a so-called potential function $\varphi(\mathbf{r}, t)$ as $\mathbf{u}=\nabla \varphi$. As a consequence, the continuity equation becomes a Laplace equation of velocity potential $\varphi$, which is also the governing equation for the potential flow, and is given by Eq. (5):

$$
\Delta \varphi=0
$$

Eq. (5) could be transformed into the following boundary integral equation by Green's third identity:

$$
\alpha_{u}\left(\mathbf{r}_{c o l}, t\right) \varphi\left(\mathbf{r}_{c o l}, t\right)=\oint\left[\frac{\partial \ln \left(\left|\mathbf{r}_{i n t}-\mathbf{r}_{c o l}\right|\right)}{\partial n} \varphi\left(\mathbf{r}_{i n t}, t\right)-\frac{\partial \varphi\left(\mathbf{r}_{i n t}, t\right)}{\partial n} \ln \left(\left|\mathbf{r}_{i n t}-\mathbf{r}_{c o l}\right|\right)\right] d l,
$$

where $\mathbf{r}_{i n t}$ and $\mathbf{r}_{c o l}$ are the position vectors of integration and collocation points on the boundary, respectively. And the value of $\alpha_{u}\left(\mathbf{r}_{c o l}, t\right)$ depends on the position of collocation point. If it is inside the computational domain, $\alpha_{u}\left(\mathbf{r}_{c o l}, t\right)=2 \pi$; if it is on the boundary, the value is the opening angle (subtending towards the fluid domain) of the boundary at $\mathbf{r}_{c o l}$. For continuous boundary such as $1^{\text {st }}$ order (or higher) smooth curve or straight-line, this angle is $\alpha_{u}\left(\mathbf{r}_{c o l}, t\right)=\pi$. The direction of the normal vector $\mathbf{n}$ is pointing towards the outside of the fluid domain, as shown in Figure 1. The 
integration will be singular when the integration point passes through the collocation point on the boundary. This is handled in the Cauchy Principal Value (CPV) sense.

The boundary conditions for the integral equation (Eq. (6)) are given as follows. First, on the moveable solid boundaries such as wave-maker or other fixed wall boundaries, the following impenetrable condition is applied:

$$
\frac{\partial \varphi}{\partial n}=\left\{\begin{array}{lll}
\mathbf{V}(\mathbf{r}, t) \cdot \mathbf{n}=V_{n}, & \text { on } & \Gamma_{w m} \\
0, & \text { on } & \Gamma_{f w}
\end{array}\right.
$$

where $\mathbf{V}(\mathbf{r}, t)$ is the velocity of the point at $\mathbf{r}$ on the boundary. $\Gamma_{w m}$ and $\Gamma_{f w}$ represent the wave-maker and fixed solid boundaries respectively.

By rearranging the Bernoulli equation, the dynamic condition for potential $\varphi$ on the free surface $\Gamma_{f s}$ takes the following form:

$$
\frac{D \varphi}{D t}=\frac{1}{2}|\nabla \varphi|^{2}-g y
$$

where $\frac{D}{D t}$ is the material derivative, defined as: $\frac{D}{D t}=\frac{\partial}{\partial t}+(\nabla \varphi \cdot \nabla)$. And the dynamic condition of zero pressure on the free surface is applied in the derivation of Eq. (8). The kinetic condition on the free surface is given by:

$$
\frac{D \mathbf{r}}{D t}=\nabla \varphi
$$

This boundary integral equation Eq. (6) together with the boundary conditions Eq. (7) to (9) will be descritized and solved numerically on the whole boundary of the computational domain at each time step. More specifically, the free surface is descritized by the spline method, whereas other solid boundaries are simply represented by straight-lines. The integration of sources and dipoles on free surface and solid boundaries are different. On the solid boundaries i.e. straight-lines, the integration could be solved analytically, whereas on the free surface, the integrations are calculated by numerical quadratures. The details of the implementation could be found in (Sun et al., 2012).

After solving the unknown boundary values at each time step, the position and velocity potential of free surface are advanced into next time step. A brief illustration of the time stepping scheme for free surface are given below.

\subsubsection{The time integration for free surface}

The position and velocity potential $\varphi$ have to be advanced to next time step to initialise the solving of the boundary integral equation. Instead of the traditional Runge-Kutta or Adams-Bashforth-Moulton methods, a truncated Taylor expansion formulation (Grilli and Subramanya, 1996; Sun et al., 2012) is employed in this study:

$$
\begin{gathered}
\mathbf{r}(t+\Delta t)=\mathbf{r}(t)+\sum_{k=1}^{m} \frac{\Delta t^{k}}{k !} \frac{D^{k} \mathbf{r}(t)}{D t^{k}}+O\left[(\Delta t)^{m+1}\right] \\
\varphi(\mathbf{r}(t+\Delta t), t+\Delta t)=\varphi(\mathbf{r}(t), t)+\sum_{k=1}^{m} \frac{\Delta t^{k}}{k !} \frac{D^{k} \varphi(\mathbf{r}(t), t)}{D t^{k}}+O\left[(\Delta t)^{m+1}\right]
\end{gathered}
$$

The first and second order material derivatives in Eq. (10) and (11) are expressed in the local coordinate system $\mathbf{n}-\boldsymbol{\tau}$ as shown in Figure 1. After some mathematical operations, the forms of these 
derivatives can be expressed as:

$$
\begin{gathered}
\frac{D \mathbf{r}}{D t}=\left[\frac{\partial \varphi}{\partial \tau} \frac{d x}{d \tau}-\frac{\partial \varphi}{\partial n} \frac{d y}{d \tau}, \frac{\partial \varphi}{\partial n} \frac{d x}{d \tau}+\frac{\partial \varphi}{\partial \tau} \frac{d y}{d \tau}\right] \\
\frac{D^{2} \mathbf{r}}{D t^{2}}=\left[\left(\frac{\partial^{2} \varphi}{\partial t \partial \tau}+\frac{\partial \varphi}{\partial \tau} \frac{\partial^{2} \varphi}{\partial s^{2}}+\frac{\partial \varphi}{\partial n} \frac{\partial^{2} \varphi}{\partial n \partial \tau}\right) \frac{d x}{d \tau}\right. \\
+\left(\frac{\partial \varphi}{\partial n} \frac{\partial^{2} \varphi}{\partial \tau^{2}}-\frac{\partial \varphi}{\partial \tau} \frac{\partial^{2} \varphi}{\partial \tau^{2}}-\frac{\partial^{2} \varphi}{\partial t \partial n}-\left(\frac{\partial^{2} y}{\partial \tau^{2}} \frac{\partial x}{\partial \tau}-\frac{\partial^{2} x}{\partial \tau^{2}} \frac{\partial y}{\partial \tau}\right)|\nabla \varphi|^{2}\right) \frac{d y}{d \tau} \\
,\left(\frac{\partial^{2} \varphi}{\partial t \partial \tau}+\frac{\partial \varphi}{\partial \tau} \frac{\partial^{2} \varphi}{\partial \tau^{2}}+\frac{\partial \varphi}{\partial n} \frac{\partial^{2} \varphi}{\partial n \partial \tau}\right) \frac{d y}{d \tau} \\
\left.+\left(\frac{\partial^{2} \varphi}{\partial t \partial n}-\frac{\partial \varphi}{\partial n} \frac{\partial^{2} \varphi}{\partial \tau^{2}}+\frac{\partial \varphi}{\partial \tau} \frac{\partial^{2} \varphi}{\partial n \partial \tau}+\left(\frac{\partial^{2} y}{\partial \tau^{2}} \frac{\partial x}{\partial \tau}-\frac{\partial^{2} x}{\partial \tau^{2}} \frac{\partial y}{\partial \tau}\right)|\nabla \varphi|^{2}\right) \frac{d x}{d \tau}\right] \\
\frac{D^{2} \varphi}{D t^{2}}=\frac{\partial \varphi}{\partial \tau} \frac{\partial^{2} \varphi}{\partial t \partial \tau}+\frac{\partial \varphi}{\partial n} \frac{\partial^{2} \varphi}{\partial t \partial n}+\frac{\partial \varphi}{\partial \tau}\left(\frac{\partial \varphi}{\partial \tau} \frac{\partial^{2} \varphi}{\partial \tau^{2}}+\frac{\partial \varphi}{\partial n} \frac{\partial^{2} \varphi}{\partial n \partial \tau}\right)-\frac{\partial \varphi}{\partial n}\left(\frac{\partial \varphi}{\partial n} \frac{\partial^{2} \varphi}{\partial \tau^{2}}-\frac{\partial \varphi}{\partial \tau} \frac{\partial^{2} \varphi}{\partial n \partial \tau}\right) \\
+\frac{\partial \varphi}{\partial n}|\nabla \varphi|^{2}\left(\frac{d^{2} y}{d \tau^{2}} \frac{d x}{d \tau}-\frac{d^{2} x}{d \tau^{2}} \frac{d y}{d \tau}\right)-g\left(\frac{\partial \varphi}{\partial n} \frac{d x}{d \tau}+\frac{\partial \varphi}{\partial \tau} \frac{d y}{d \tau}\right)
\end{gathered}
$$

In Eq. (12) to (14), the tangent spatial derivatives of $\varphi$ and $\frac{\partial \varphi}{\partial n}$, such as $\frac{\partial \varphi}{\partial \tau}$ and $\frac{\partial^{2} \varphi}{\partial n \tau}$, can be calculated directly from the $\varphi$ and $\frac{\partial \varphi}{\partial n}$ distributions provided by the boundary conditions Eq. (7) to (9). The time derivatives such as $\frac{\partial \varphi}{\partial t}$ and $\frac{\partial^{2} \varphi}{\partial n \partial t}$ are calculated in the following manner: first, taking the time derivatives on both sides of Eq. (6), then a new boundary integral equation about $\frac{\partial \varphi}{\partial t}$ and $\frac{\partial \varphi}{\partial n \partial t}$ can be obtained and then solved in the same way for Eq. (6). The boundary conditions for this new boundary integral equation are as follows:

For the free surface, the value of $\frac{\partial \varphi}{\partial t}$ could be obtained by Bernoulli's equation.

$$
\frac{\partial \varphi}{\partial t}=-\frac{1}{2}|\nabla \varphi|^{2}-g y
$$

On the wave-maker or other fixed wall boundaries, the time derivative of Eq. (7) is applied:

$$
\frac{\partial^{2} \varphi}{\partial n \partial t}=\left\{\begin{array}{lll}
\frac{\partial(\mathbf{V}(\mathbf{r}, t) \cdot \mathbf{n})}{\partial t}, & \text { on } & \Gamma_{w m} \\
0, & \text { on } & \Gamma_{f w}
\end{array}\right.
$$

For a piston type wave-maker, which will be used later in the simulation, it can be expressed as:

$$
\frac{\partial^{2} \varphi}{\partial n \partial t}=-\dot{\mathbf{V}}-\mathbf{V} \frac{\partial^{2} \varphi}{\partial \tau^{2}}
$$

\section{Weak coupling between BEM and MPS}

For many free surface flow problems in marine engineering, the violent flow phenomena such as wave breaking, is only restricted to a small area which is close to the ship or other marine structures. For this kind of cases, the majority of the flow could be simply described by potential flow theory, which is more efficient than the direct simulation using Navier-Stokes equations. 


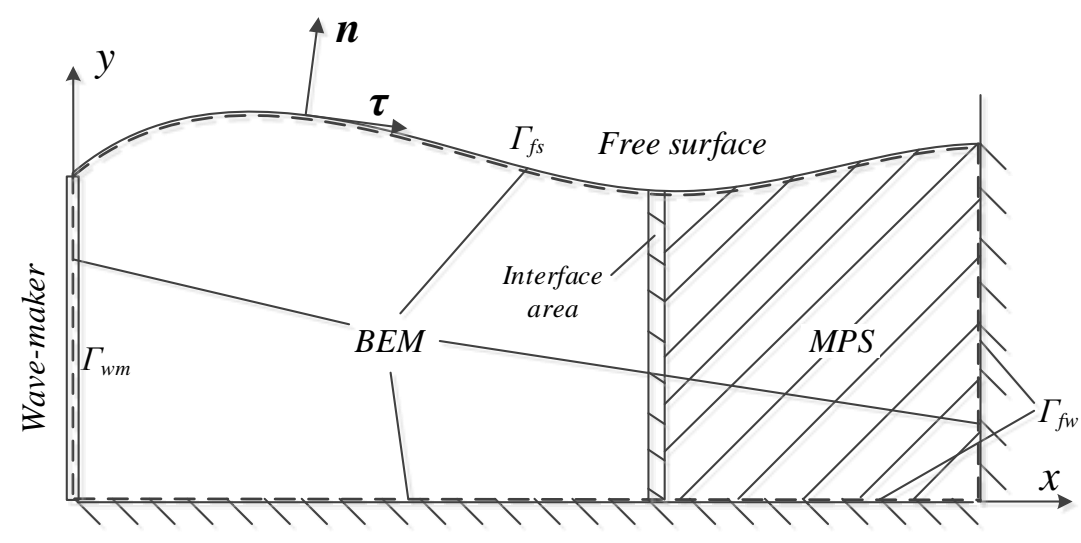

Figure 1: Computational domain layout and coordinates system for weak coupling between BEM and MPS

In this section, the $2 D$ weak coupling scheme between the potential flow based Boundary Element Method (BEM) and the proposed modified MPS method, as shown in Figure 1, is investigated.

Compared with the strong coupling case, the main feature of weak coupling strategy is that the information will only be feed from one solver to another, and there is no mutual exchange. In this particular case, this means the BEM solver, which covers the whole computational domain as indicated by the dashed line on the whole boundaries in Figure 1, will provide all the necessary boundary information (e.g. velocity, pressure) to the MPS area. And the MPS solver do not provide feedback to BEM solver. This way of coupling is easier to implement than the strong coupling case, and it will also avoid the stability issue that is introduced by the exchanging of information in strong coupling. However, it will not be as capable as the strong coupling for the situation where significant interaction occurs between potential flow and Navier-Stokes domains. Nevertheless, as mentioned in the beginning of this chapter, for many cases where the violent free surface deformation only occurs in a restricted area, this simple way of coupling is a more efficient choice. The details of the weak coupling approach is described as follows:

As shown in Figure 1, the area covered by shadow is modelled by MPS method. The whole computational domain is discretised by BEM nodes. More specifically, as aforementioned, the whole domain will be initially modelled by BEM method up to the time when the wave is about to break (but not breaking yet). The MPS area will also be running from the beginning by taking the pressure and velocity results of BEM as boundary condition at the interface area (i.e. overlapping area shown in Figure 1). When the wave modeled by BEM is about to break, the BEM simulation will stop and all the boundary values remain the same. The MPS simulation will continue for the wave structure impact calculation. Considering that the interface area is chosen to be far away from the impacting area, the pressure and velocity of this area will not vary a lot for the whole "post-breaking" period (which is close to the state of calm water). As a result, using the "pre-breaking" BEM value to impose the MPS boundary condition on interface area for post-breaking duration will not affect the results significantly.

At the interface between MPS and BEM domains, the pressure and velocity for MPS particles are calculated from the BEM solution. These interface particles also move based on the calculated velocity from BEM. 


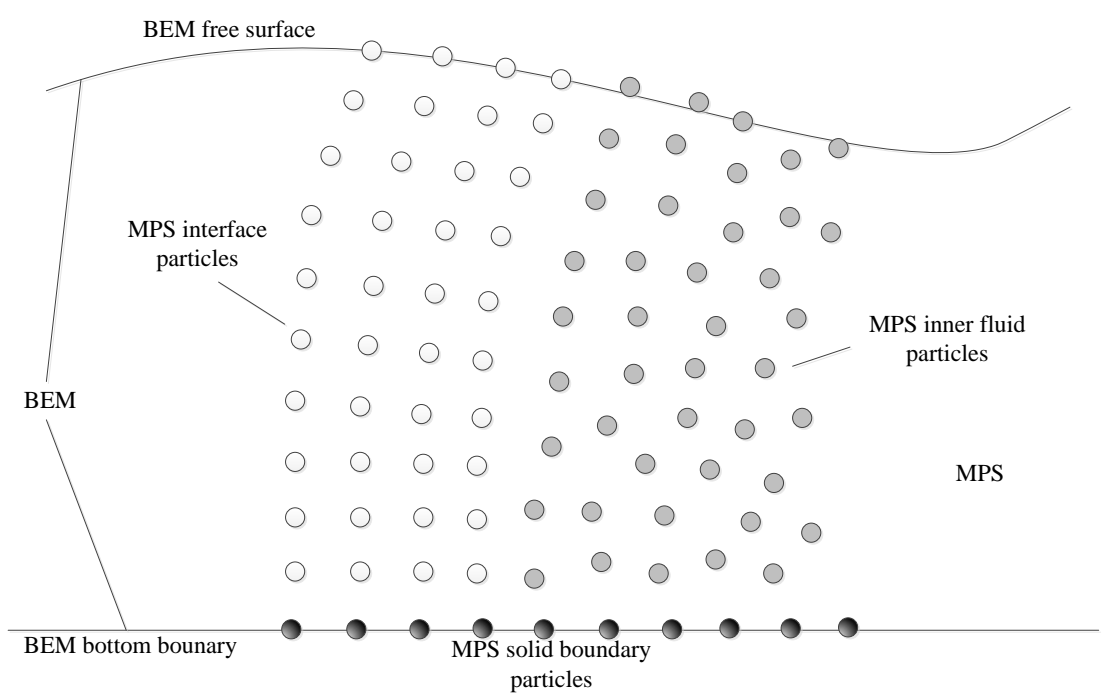

Figure 2: Sketch of the BEM-MPS interface

The particle arrangements at the interface area are shown in Figure 2. The first four columns of MPS particles are treated as the interface particles. Their velocity are calculated based on the values of $\varphi$ and $\frac{\partial \varphi}{\partial n}$ on the BEM boundaries. More specifically, the velocity is calculated by taking gradient operation on both side of Eq. (6) as follows:

$$
\begin{aligned}
\alpha_{u}\left(\mathbf{r}_{c o l}, t\right) \nabla_{c o l} \varphi\left(\mathbf{r}_{c o l}, t\right)=\oint & {\left[\nabla_{c o l}\left(\frac{\partial \ln \left(\left|\mathbf{r}_{i n t}-\mathbf{r}_{c o l}\right|\right)}{\partial n}\right) \varphi\left(\mathbf{r}_{i n t}, t\right)\right.} \\
& \left.-\frac{\partial \varphi\left(\mathbf{r}_{i n t}, t\right)}{\partial n} \nabla_{c o l}\left(\ln \left(\left|\mathbf{r}_{i n t}-\mathbf{r}_{c o l}\right|\right)\right)\right] d l,
\end{aligned}
$$

where $\nabla_{c o l}$ indicates that the gradient operation is with respect to the coordinates of the collocation point $\mathbf{r}_{c o l}$. On the straight-line boundaries such as wave-maker or fixed solid walls, the integration on the right hand side could be calculated analytically by the formulation provided in Ref (He and Dai, 1991). For the curved free surface, the "Quasi-spline" element (between two adjacent nodes) is further divided into several "sub-elements", which are simply represented by straight-lines. And then the integrations are calculated on these "sub-elements" by the same analytical approach used for straight-line boundaries.

The pressure of the interface MPS particles are calculated based on Bernoulli's equation as:

$$
p=-\left(\frac{\partial \varphi}{\partial t}+\frac{1}{2}|\nabla \varphi|^{2}+g y\right) \rho
$$

The value of $\frac{\partial \varphi}{\partial t}$ is calculated directly from the solution of the additional integral equation for $\frac{\partial \varphi}{\partial t}$ and $\frac{\partial \varphi^{2}}{\partial n \partial t}$, as shown in Section 2.2.2. 


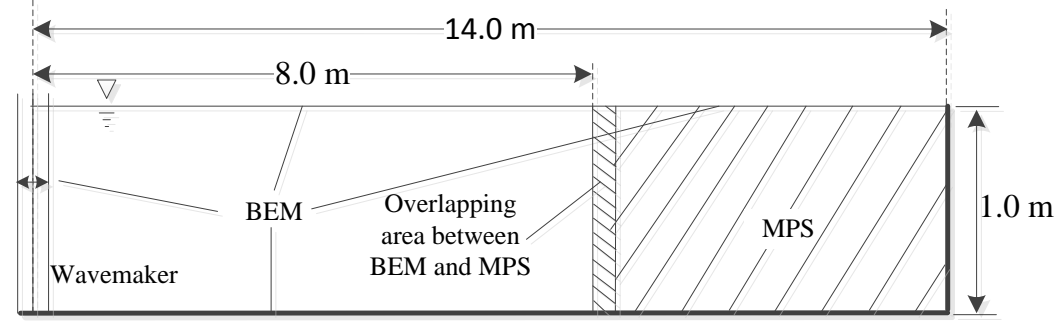

Figure 3: Sketch of the regular wave simulation by coupling BEM-MPS

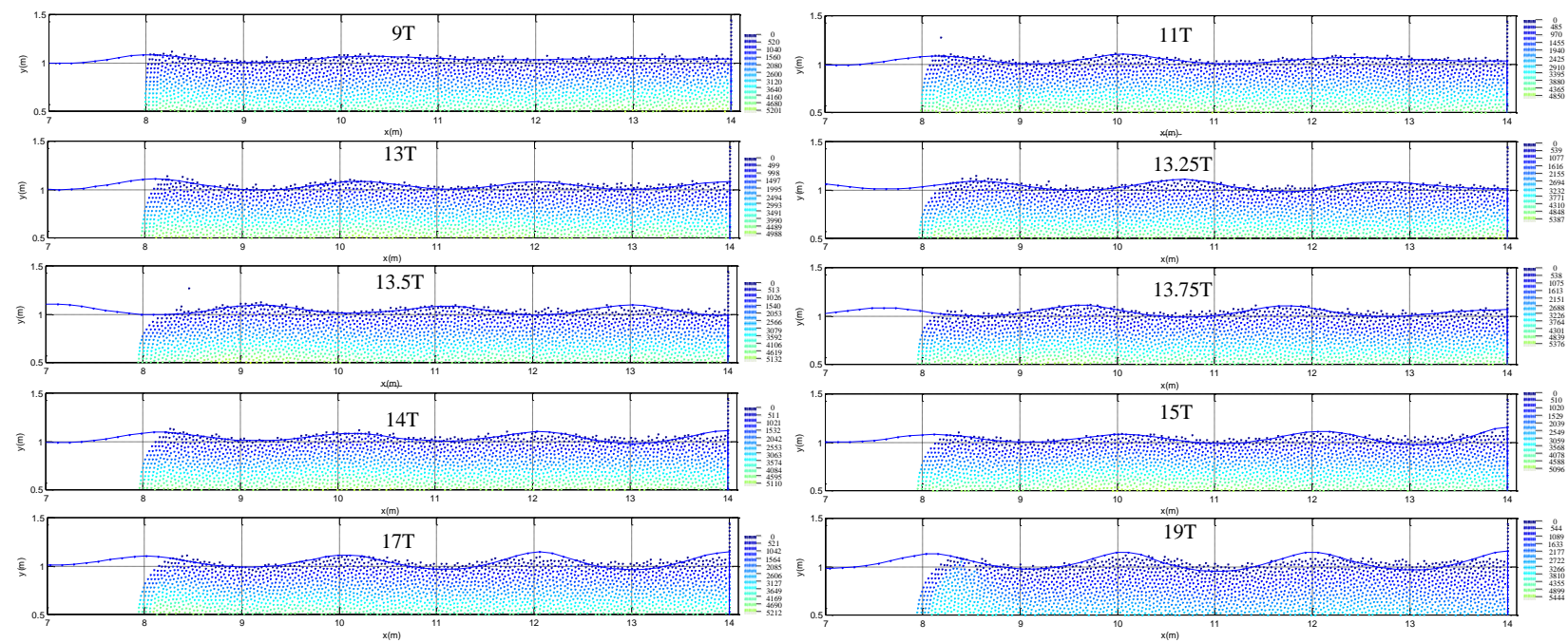

Figure 4: Pressure contour and free surface profile at some typical time instant

\section{Results and Discussion}

\subsection{Validation by regular wave propagation}

In order to validate the concept of the proposed weak coupling scheme, the case of regular wave propagation is simulated. The geometric configuration of the problem is shown in Figure 3. A piston type wave maker is used to generate the regular wave. The circular frequency and wave amplitude (half wave height) are 5.54 and $0.05 \mathrm{~m}$, respectively.

For the MPS part, the space is descritized by particle interval of $0.04 \mathrm{~m}$, which results in 3750 fluid particles (4124 particles in total). On the BEM free surface (which covers the whole free surface area as explained before), in total of 116 nodes are used for descritization, which means that there are about 16 nodes along each wave length for this wave condition.

The pressure contour of the MPS part and the free surface shapes of the BEM part are compared in Figure 4, where $T$ is the period of the wave. The length of the tank is roughly 7 times of the wave length in this case, consequently a standing wave is gradually generated due to the wave reflection by the wall, and as a result, the wave amplitude is getting larger. The BEM result, which is the blue solid line, is generally matching well with the MPS free surface shapes at various typical time instants as shown in Figure 4. However, although the general shape of the wave profile is consistent with BEM 


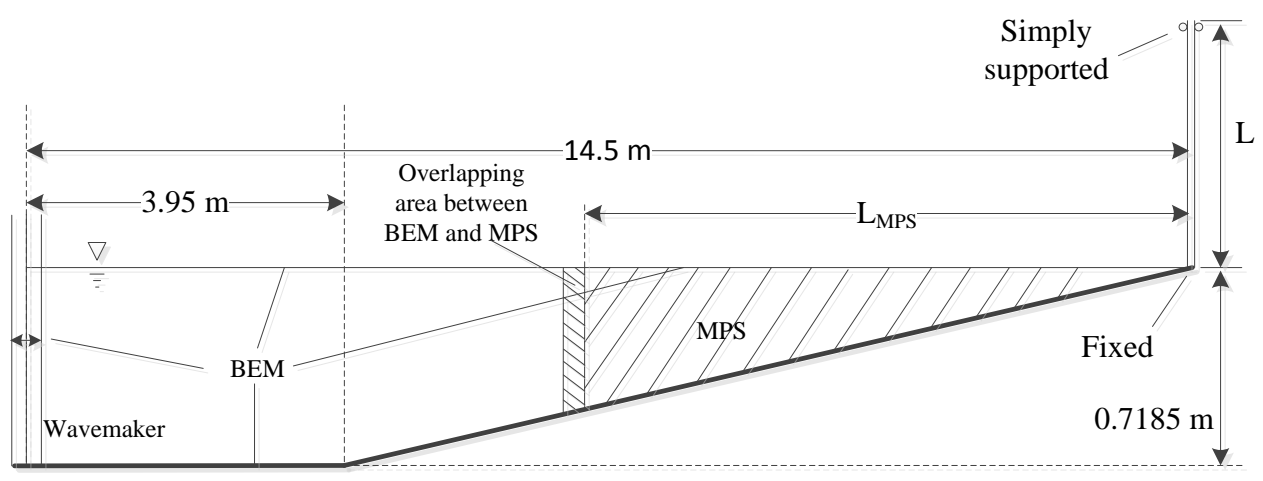

Figure 5: Sketch of the solitary wave climbing small slope ramp by coupling BEM and MPS.

result, the MPS free surface shape becomes less smooth as the wave amplitude getting larger (e.g. the $19 T$ time instant in Figure 4).

Overall this example proves that the data exchanging scheme of the weak coupling scheme is capable of providing accurate boundary condition (from BEM results) to enable the running of MPS solver.

\subsection{Breaking wave impacting flexible wall}

In order to further test the weak coupling scheme, the process of a violent breaking wave interaction with vertical flexible wall was simulated (Sriram and Ma.Q.W., 2012; Kimmoun et al., 2009). The problem set-up, results and discussions are presented in this section.

As shown in Figure 5, a wave tank with piston-type wave-maker is used to generate solitary wave, which then runs up on a gentle slop. A flexible vertical plate with length of $1 \mathrm{~m}$ is mounted at the end of the tank and simply supported at $0.88 \mathrm{~s}$ from the plate bottom, with the top free of restriction. The width of the plate is $0.65 \mathrm{~m}$. The density and Young's modulus of the elastic plate are $1190 \mathrm{~kg} / \mathrm{m}^{3}$ and $3250 M P a$. The geometric dimensions of the wave tank is depicted in Figure 5. The method mentioned in (Grilli and Subramanya, 1996) is used to generated the solitary wave with amplitude of $0.08 \mathrm{~m}$. Both experimental (Kimmoun et al., 2009) and numerical (Sriram and Ma.Q.W., 2012) studies of this problem have been conducted. Comparison between these previous and the current results will be presented later.

For the case reported below, the length of the MPS domain, including the overlapping area, is chosen to be $7.5 \mathrm{~m}$. The first four columns of particles will be treated as interface particles whose pressure and velocity are determined by the BEM solver. The initial particle distance is chosen to be $0.0075 \mathrm{~m}$, which leads to a total of 33772 fluid particles. If the whole domain is discritized by MPS method, the fluid particle used for this geometry would be more than double of the current number. The BEM free surface is descritized by 85 nodes. The time step is controlled by CFL condition with initial value of $0.0015 s$.

The flexible deformation of the plate is modeled as a beam and computed by modal superposition approach (Paz and Leigh, 2004). And the first 3 principle modes are used in the simulation. The iterative procedure that mentioned in (Sun et al., 2016) is used for the interaction between fluid solvers and structure solver. The time history of the plate deformation at $0.3623 m$ from the plate 


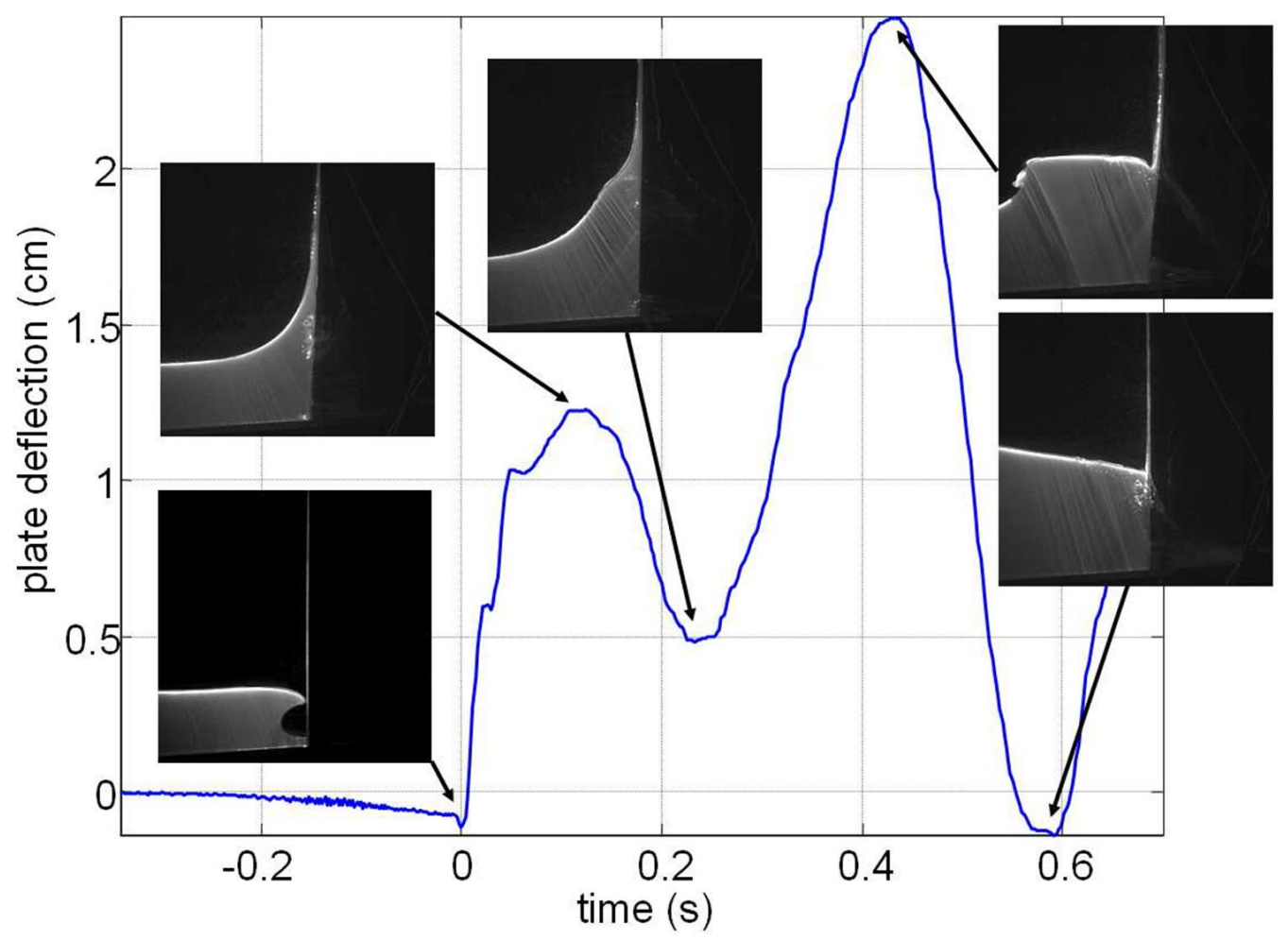

Figure 6: Experimental results of the plate deformation and wave profiles at some typical time instants

bottom and the wave profiles of some typical time instants, from the experiment (Kimmoun et al., 2009) and the proposed BEM-MPS model are shown in Figure 6 and Figure 7, respectively. The major trend of these two deflection time history curves match each other very well. The two peaks of the deformation occur at about $0.12 \mathrm{~s}$ and $0.43 \mathrm{~s}$ in the experiment, whereas in the numerical result the times for these two peaks are $0.10 \mathrm{~s}$ and $0.414 \mathrm{~s}$. The wave profiles for the corresponding time instants are very similar as well.

The comparison of deflection time history at the same position between experiment, the numerical approach (MLPG_R) (Sriram and Ma.Q.W., 2012) and the proposed BEM-MPS coupling model is shown in Figure 8. As discussed in (Sriram and Ma.Q.W., 2012), the reason of the negative deflection before the impact of the breaking wave in the experimental result is unclear. This may due to the way of mounting the plate at the bottom. Besides that, although the main trends of these three curves are quite similar, there are inevitably some discrepancies between them. The first peak values of both numerical results are about $20 \%$ lower than the experimental one. For the second peak, the value from proposed model is slightly lower (about 10\%) than the experiment and MLPG_R. The peaks and troughs from the proposed method are also slightly shifted compared with the results from others. The most reasonable explanation that the authors can give is as follows: up until the wave-breaking, the BEM computation stops and the MPS solver continues the post-breaking simulation. Therefore in the interface area, the MPS will not get new update of the flow state, though during this time the flow change are expected to be very small in this area (which is the base of this coupling scheme). Nevertheless, this way of weak coupling is considered to be responsible for the slight mismatch aforementioned. Apart from that, the differences between numerical and experimental results could be due to various reasons such as the leakage of water from the wave-maker and plate in the experiment (Sriram and Ma.Q.W., 2012). 


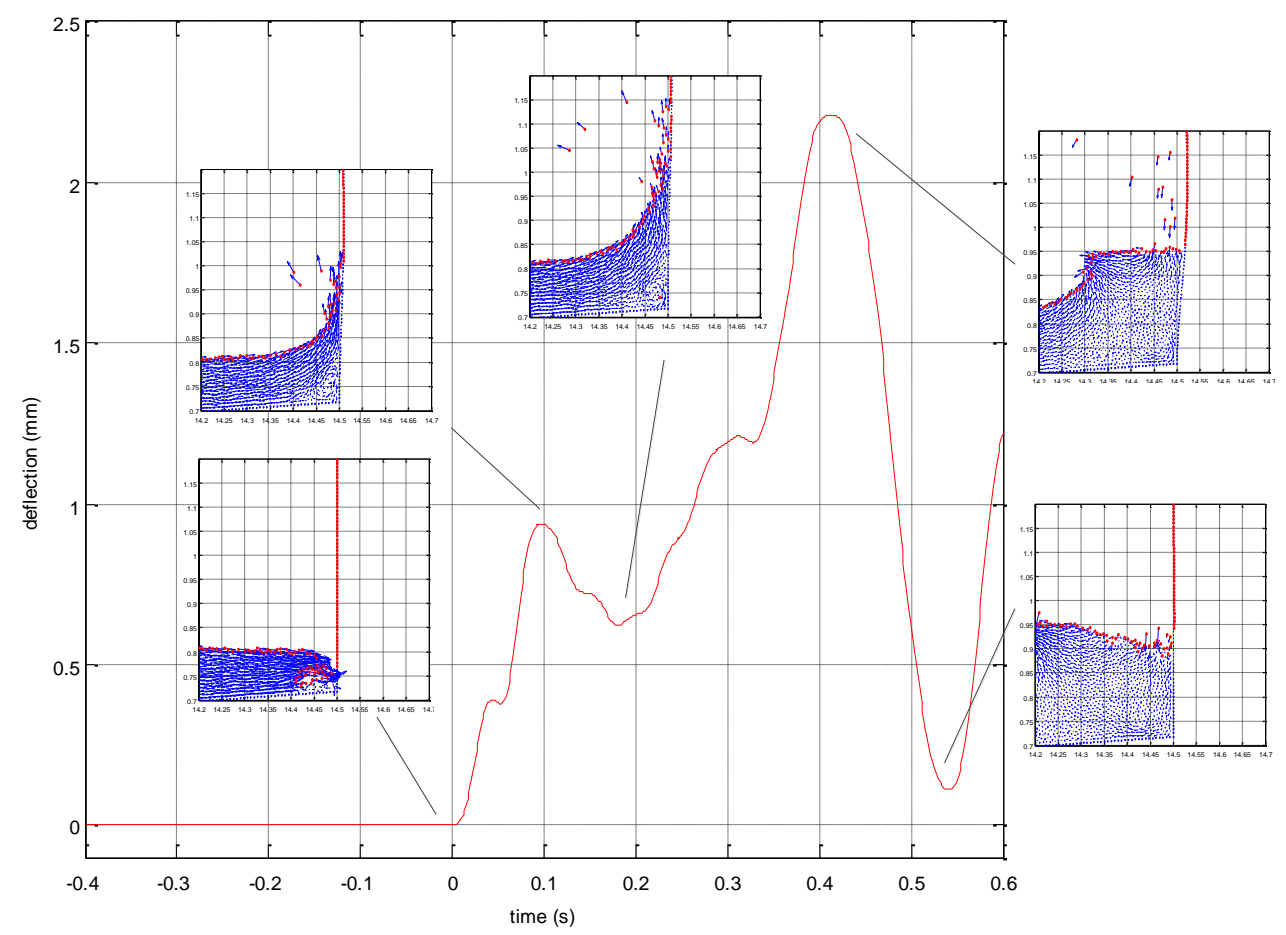

Figure 7: Coupled BEM-MPS results of the plate deformation and wave profiles at some typical time instants

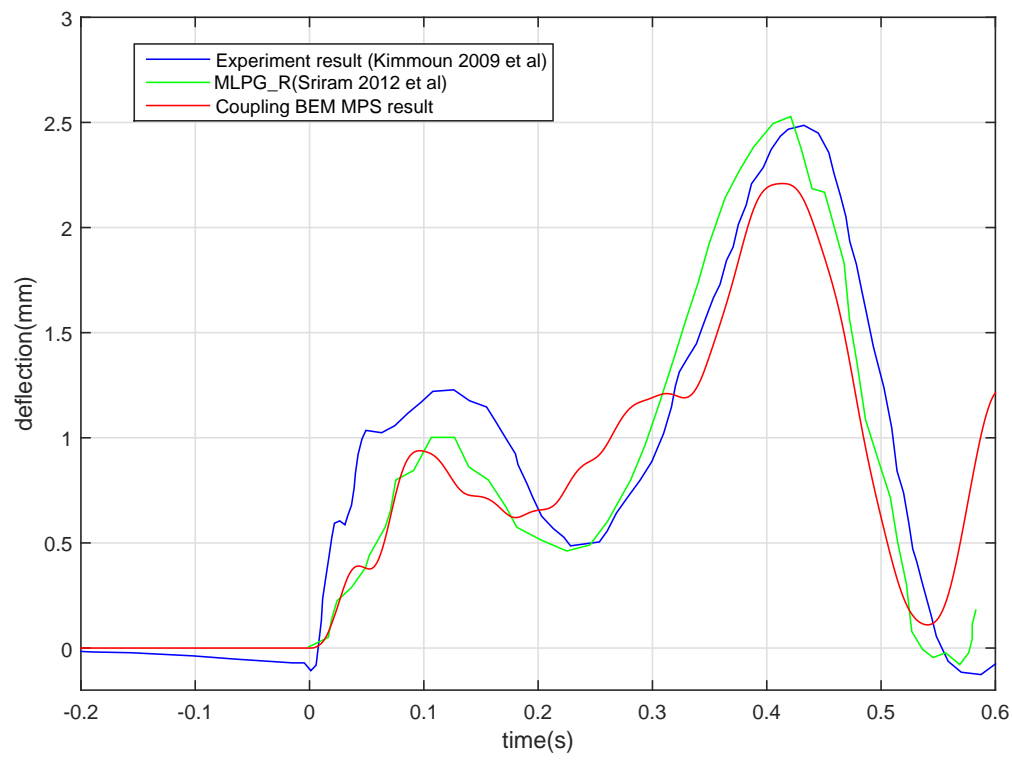

Figure 8: Comparison of the plate deflection at $0.3623 \mathrm{~m}$ from the plate bottom between experiment (Kimmoun et al., 2009), MLPG_R (Sriram and Ma.Q.W., 2012) and proposed BEM-MPS coupling 

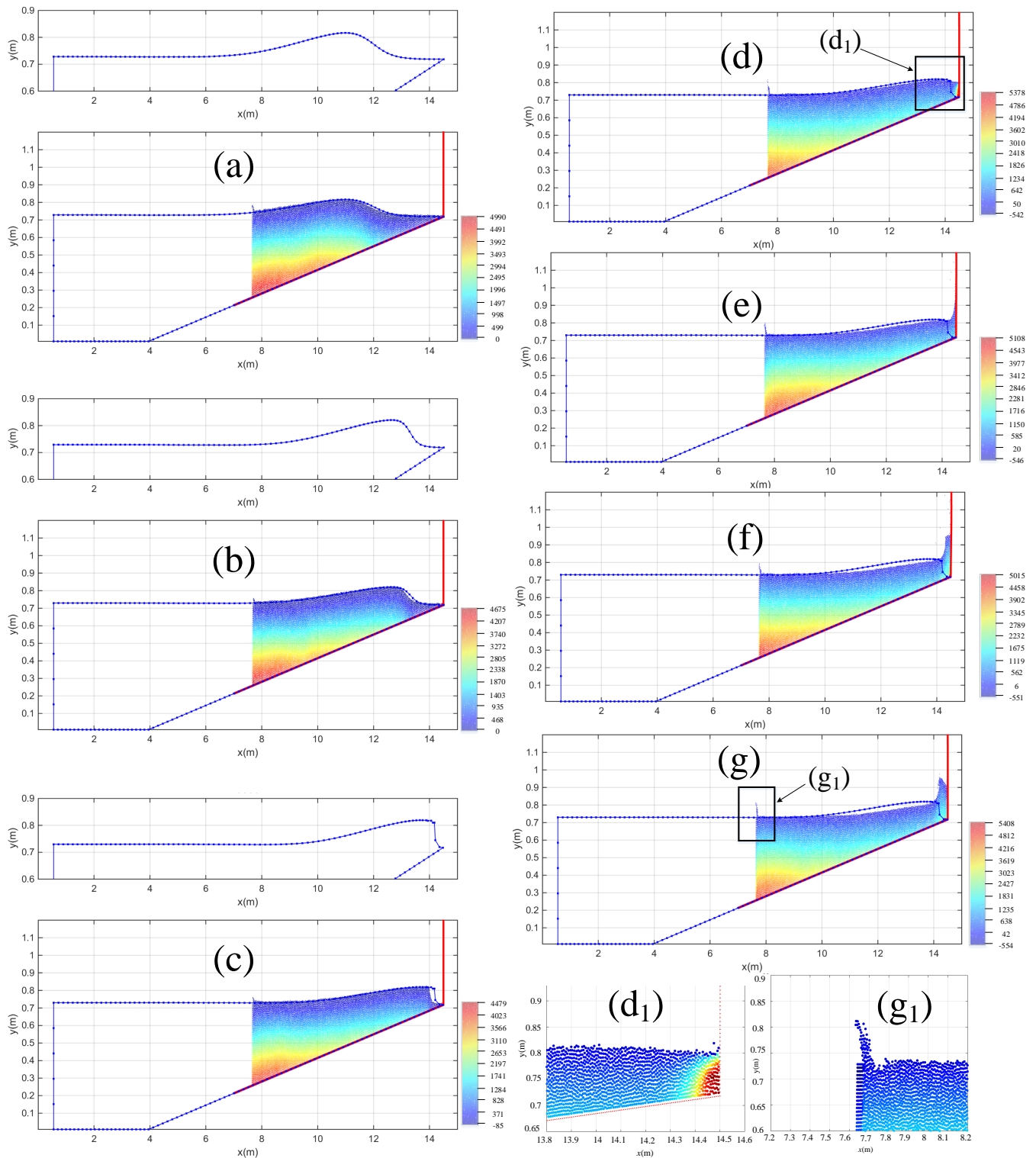

Figure 9: Wave profiles and the pressure contour of the BEM and MPS simulation at various typical time instants

In terms of efficiency, in comparison to another particle method, i.e. MLPG_R (Sriram and Ma.Q.W., 2012), where the whole domain is discretised by a similar resolution with 86670 fluid particles (compared with 33772 fluid particles in this simulation), this problem is solved more efficiently by the proposed BEM-MPS coupling approach with similar accuracy achieved.

The BEM wave profiles and MPS pressure contours are shown in Figure 9. The BEM simulation stops at $t=8.821 s$, i.e. (c) or (d) in Figure 9 as explained before, so the BEM wave profile remains the same from Figure 9 (d) to $(\mathrm{g})$. As is shown, the pressure distribution from the MPS simulation is smooth. In Figure 9, $\left(\mathrm{d}_{1}\right)$ highlights the pressure distribution when the wave impacts the plate. It is worth mentioning that a small "jet-like" flow has been developed as highlighted by Figure $9\left(\mathrm{~g}_{1}\right)$. The pressure in that area becomes slightly negative as can be seen from the legend. This physically unreasonable phenomena could be due to the fact that MPS field gradually starts to flow backward while the interface particles remain the status when BEM simulation stops (i.e. $t=8.821 s$ in $(\mathrm{c}$ ) or 
(d) of Figure 9), so the fluid particles will be "squeezed out" (due to negative pressure in that area) a bit more near the interface area. However as shown in the above results, the main flow characteristics near the plate and the flexible plate deformation have both reached an acceptable accuracy compared to the corresponding experimental or the numerical scheme MLPG_R, which is the major concern of this simulation. This means if we consider the balance between the efficiency improvement and the accuracy level it can get, this weak coupling between BEM and MPS is capable to simulate this kind of locally violent flow problems. But obviously a strong coupling, i.e. the BEM solver only covers part of the computational domain and depends on the information from MPS solver at interface, would be an advanced and better choice in the future.

\section{Conclusion}

In order to improve the computational efficiency of MPS method, the weak coupling scheme between BEM and modified MPS is proposed and implemented for the problems where the violent free surface deformation only occurs in a constrained area.

In this weak coupling scheme, the BEM covers the whole computational domain and runs until the time when free surface is about to break. The MPS solver, which also starts from the beginning but only occupies the rapid fluid structure interaction area, will continue the simulation for the post-breaking phase. The MPS solver is enabled by the information from BEM solver (i.e. velocity and pressure), and there is no feedback from MPS to BEM. The MPS will continue to use the BEM information at the time before BEM solver stops, since the changing rate of the flow in the BEM-MPS interface area is small enough. The computational time could be reduced, since the BEM is far more efficient than MPS for the same computational area.

The weak coupling scheme between BEM and modified MPS is validated by two problems, i.e. the propagation of regular wave and the breaking solitary wave impacting flexible wall. The BEMMPS coupled fluid solver interacts with structural solver (which is just typical modal superposition model (Paz and Leigh, 2004)) in an iterative manner. The numerical results are compared with experimental results published in the literature, and reasonably good agreement is achieved. In these two cases, more than half of the computational domain is replaced by BEM, so the computational efficiency is significantly improved. However, there are some obvious limitations about this weak coupling scheme due to the fact that there is no feedback of flow information from MPS solver, such as the non-physical flow phenomena around the interface area mentioned in Section 4.2. This kind of problems should ideally be solvable by employing the strong coupling approach, which would be considered in future work.

\section{Acknowledgements}

The research is co-sponsored by Lloyd's Register, University of Southampton and China Scholarship Council. The authors would like to express sincere thanks for their support

\section{References}

C.F.Janssen, S.T.Grilli, and M.Krafczyk (2010). Modeling of wave breaking and wavestructure interactions by coupling of fully nonlinear potential flow and latticeboltzmann models. In 20th International Offshore Polar Engineering Conference, Beijing,China. 
Chorin, A. (1967). A numerical method for solving incompressible viscous flow problems. Journal of Computational Physics, 2:12-26.

C.Lachaume, B.Biausser, S.T.Grilli, P.Fraunie, and S.Guignard (2003). Modeling of breaking and post-breaking waves on slopes by coupling of BEM and VOF methods. In 13th International Offshore Polar Engineering Conference, Honolulu,HI, USA.

Colicchio, G., Greco, M., and Faltinsen, O. M. (2006). A BEM-level set domain-decomposition strategy for non-linear and fragmented interfacial flows. International Journal for Numerical Methods in Engineering, 67(10):1385-1419.

Duan, G. and Chen, B. (2015). Comparison of parallel solvers for moving particle semi-implicit method. Engineering Computations, 32(3):834-862.

Grilli, S. (2008). On the development and application of hybrid numerical models in nonlinear free surface hydrodynamics. In 8th International Conference on Hydrodynamics, Nantes, France.

Grilli, S. and Subramanya, R. (1996). Numerical modeling of wave breaking induced by fixed or moving boundaries. Computational Mechanics, 17(6):374-391.

He, W. and Dai, Y. (1991). On the calculation of induced potentials and velocities by some most used singularity line segments (in chinese). Journal of Harbin Shipbuilding Engineering Institute, 12(2):139-147.

Kassiotis, C., Ferrand, M., Violeau, D., and Rogers, B. (2011). Coupling SPH with a 1-D boussinesqtype wave model. In The 6th International SPHERIC Workshop, Hamburg, Germany.

Kimmoun, O., Malenica, ., and Scolan, Y.-M. (2009). Fluid structure interactions occuring at a flexible vertical wall impacted by a breaking wave. In International Offshore and Polar Engineering(ISOPE), Osaka, Japan.

Koshizuka, S. and Oka, Y. (1996). Moving-particle semi-implicit method for fragmentation of incompressible fluid. Nuclear Science and Engineering, 123:421-434.

Li, S. and Liu, W. K. (2002). Meshfree and particle methods and their applications. Applied Mechanics Reviews, 55(1):1.

Li, S. f. and Liu, W. k. (2007). Meshfree Particle Methods. Springer.

Paz, M. and Leigh, W. (2004). Structural Dynamics: Theory and Computation (5th Edition). Kluwer Academic Publishers.

Saad, Y. and Schultz, M. H. (1986). Generalized minimal residual algorithm for solving nonsymmetric linear systems. SIAM J. SCI. STAT. COMPUT., 7(3):856-869.

Shibata, K., Koshizuka, S., Tamai, T., and Murozono, K. (2012). Overlapping particle techniques and application on green water on deck. In International Conference on Violent Flows, Nantes, France.

Sriram, V., Ma, Q. W., and Schlurmann, T. (2014). A hybrid method for modelling two dimensional non-breaking and breaking waves. Journal of Computational Physics, 272:429-454.

Sriram, V. and Ma.Q.W. (2012). Improved MLPG_R method for simulating 2d interaction between violent waves and elastic structures. Journal of Computational Physics, 231(22):7650-7670.

Sueyoshi, M., Kihara, H., and Kashiwagi, M. (2007). A hybrid technique using particle and boundaryelement methods for wave-body interaction problems. 
Sun, Z., Djidjeli, K., Xing, J. T., and Cheng, F. (2015). Modified MPS method for the 2d fluid structure interaction problems with free surface. Computers and Fluids, 122:47-65.

Sun, Z., Djidjeli, K., Xing, J. T., and Cheng, F. (2016). Coupled MPS-modal superposition method for $2 \mathrm{~d}$ nonlinear fluidstructure interaction problems with free surface. Journal of Fluids and Structures, 61:295-323.

Sun, Z., Pang, Y., and Li, H. (2012). Two dimensional fully nonlinear numerical wave tank based on the BEM. Journal of Marine Science and Application, 11(4):437-446.

Xing, J. T. (2016). Developments of numerical methods for linear and nonlinear fuid-solid interaction dynamics with applications. Advances in Mechanics, 46(2):95-138.

Zhang, X., Song, Z. K., and Lu, M. W. (2003). Research progress and application of meshless method. Chinese Journal of Computational Mechanics, 20(6). 